\title{
Analysis of Range-Based Node Location Algorithm in WSN
}

\author{
Xiaojun Liu ${ }^{1, a}$ and Jianyu Wang ${ }^{1}$ \\ ${ }^{1}$ School of Transportation Huanggang Normal University, Hubei Huanggang, China \\ awhutliuxiaojun@126.com
}

Keywords: TOA(Time of Arrival); TDOA (Time Difference of Arrival); AOA((Angle of Arrival); Received Signa1 Strength Indicator (RSSI)

\begin{abstract}
This paper describes the basic concepts of node localization of wireless sensor networks (WSN), commonly used terms, localization algorithm classification and localization calculation methods. Focus on the description of several location algorithms based on, and analyzes the pros and cons of each algorithm, pointed out that the performance of the various algorithms and the scope of application.
\end{abstract}

\section{Introduction}

In wireless sensor network (WSN) in practical applications, the location information of sensor nodes has become one of the essential information in the network, many applications once lost the location of nodes, the network will become useless, so the sensor network node positioning technology has become an important topic for many scientists[1]. There is many the algorithms in the implementation process of localization, according to different standards have different classification methods. The most common classification is based on the distance (Range-Based) localization algorithm and range free localization algorithm (Range-Free). If the algorithm needs to know the absolute distance between unknown nodes and reference nodes and beacon nodes, and then calculates the unknown node coordinate information, this positioning method can be called range-based localization algorithm.

\section{Background}

Knowledge in sensor networks, in order to achieve positioning, nodes randomly spread mainly two: Beacon node and Unknown node. Usually known its position of node said to Beacon nodes and Beacon nodes can be through carrying the existing positioning equipment and other means to obtain its exact position, while the other nodes are called for Unknown nodes, wireless sensor networks in CITIC mark node only account for a small proportion. Unknown nodes and Beacon nodes as a point of reference, through the location information of Beacon nodes to determine its position. The nodes of the sensor network structure as shown in Fig. 1[2].

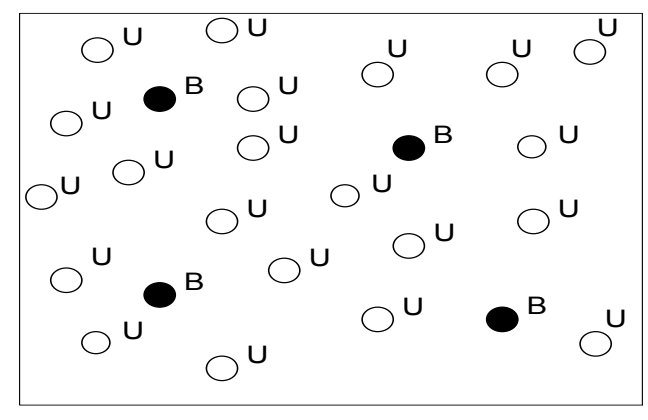

Figure 1. Beacon nodes and unknown nodes of wireless sensor network

In Fig. 1, the entire sensor network consists of four beacon nodes and a large number of unknown node compositions. Beacon node B to represent, it in the network as a whole accounted for less 
proportion. Unknown node u to represent, unknown nodes by around the beacon nodes or has achieved self localization of unknown node through certain algorithm to achieve self localization. Here are some of the commonly used terms in wireless sensor networks:

(1) Neighbor Nodes: nodes that can communicate directly with the other nodes without having to go through other nodes;

(2) Hop Count: two to achieve the communication between the nodes of the minimum number of hops required for the total number of hops;

(3) Connectivity: the number of neighbor nodes owned by a node;

(4) Hop Distance: the sum of the minimum hop distance between two nodes;

(5) Receiving Signal Propagation Time Difference (Time Difference of Arrival, TDOA): in the process of signal transmission, issued at the same time, two different frequency signals reach the same destination when because of the time difference caused by different transmission speeds.

(6) Time of Arrival (TOA): the time required for the propagation of the signal at two different nodes;

(7) Round-trip Time of Flight (RTOF): the signal from one node to another node back to the time back;

(8) Angle of Arrival (AOA): the angle between the node's own axis and the received signal;

(9) Received Signa1 Strength Indicator (RSSI): wireless signal to reach the strength value of the sensor nodes.

\section{Location Algorithm Based on Distance Measurement}

The positioning algorithm based on distance measurement to achieve more complex, first through TOA (Time of Arrival), TDOA (Time Difference of Arrival), AOA (Angle of Arrival), RSSI (Received Signa1 Strength Indicator) and absolute distance ranging technology commonly used to measure each unknown node and beacon node values, this stage is also called after the end of the ranging stage; ranging of positioning (coordinate) stage, namely the use of the node ranging stage distance or direction parameters to calculate the location of the unknown node, during which the commonly used algorithm: three edge measurement positioning method (Trilateration), multilateral localization method of triangulation method (triangulation), maximum likelihood estimation (Maximum Likelihood Method) and angle positioning method (Goniometry). Following this thesis analyzed in two phases [3-6]:

Ranging Algorithm Analysis. TOA is according to the signal propagation time calculation is measured between the node distances. TOA algorithm although the positioning precision is high, but the algorithm requires nodes precise synchronization using complex, the hardware requirements are too high and therefore less suitable to the application of wireless sensor network localization.

The TDOA is formed on the basis of the TOA algorithm. In this algorithm, using two kinds of wireless signal transmitting node and different frequency information to send a group of the same area specified, because the transmission speed of the two signals are different, so the arrival time will be different according to the receiving node. The time difference and two kinds of signal transmission speed can be calculated between the receiving node and transmitting node distance value. This method can get the accurate positioning in NLOS conditions. But the requirements of sensor nodes are equipped with RF transceiver and ultrasonic wave transceiver, thus increasing the cost. And the signal is easily affected by the environment applicable occasions, single. TDOA method is applicable to the line of sight environment, obstacles in the NLOS environment will be reflected on the acoustic wave refraction and diffraction, which would increase the ultrasonic transmission time, produced a lot of errors, and ultimately affect the accuracy of the positioning.

AOA is through Triangulation calculate the location. In the AOA algorithm, unknown nodes first to calculated with respect to a reference node of the azimuth angle, which makes the algorithm positioning performance in complex electromagnetic environment is very poor, but also improves the 
energy, which eventually led to the lifetime of the network is shortened. The WSN with low cost, the goal of low energy consumption contrary, so the measurement method of practicality is very low.

RSSI is using the channel attenuation model to realize the function of positioning nodes according to the received signal strength. The regularity of RSSI value is not strong, so there is in the measurement when the error is large, so the need for multiple anchor nodes and position, which requires a large number of anchor nodes. In practical application, signal will inevitably encounter interference during transmission, reflection, absorption and other effects, which greatly reduce the positioning accuracy. At present, the RSSI value ranging technology can be divided into two kinds: one is based on the method of pretesting of environmental information, namely in the laboratory before the start of the treatment side area for large values of RSSI test that will be a different value saved to the database, then the query and call in the laboratory. Another is the method does not require pretesting of environmental information, such as hyperbolic model, distributed iterative algorithm, and combined with the dew flooding is introduced RSSI mechanism of HCRL (hop-count-ratio based localization) algorithm.

PDOA(Phase difference of arrival) is measured by measuring the phase difference of the received signal, and the signal transmission of the round-trip time, and then calculate the distance between the signal.

NFER(Near-Field Electromagnetic Ranging) near field electromagnetic distance, is measured by the phase difference between the near field and the magnetic field to measure the distance.

Positioning Stage Algorithm Analysis. Trilateration through three known coordinates of beacon nodes and the three beacons to unknown nodes of distance information, according to the two-dimensional spatial distance formula established equations and linearization method is used to solve the unknown node location information.

As shown in Fig. 2, assuming that there are three anchor nodes B, C, a to participate in positioning, and their coordinates are $\left(x_{a}, y_{a}\right),\left(x_{b}, y_{b}\right),\left(x_{c}, y_{c}\right)$.

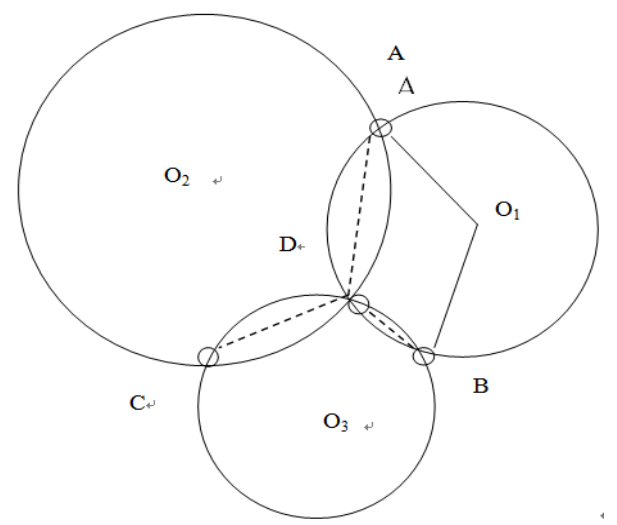

Figure 2. Trilateration

Hypothesis three anchor nodes have been measured by antenna array have node D signal direction of arrival, after determining the azimuth can be calculated angle the ADB, ADC angle, BDC angle size. Node $A, B$ and proposed $A D B$ can determine the only round $\mathrm{O}_{1}$, and $\mathrm{O}_{1}$ center coordinates $\left(\mathrm{x}_{1}, \mathrm{y}_{1}\right)$ and radius $r_{1}$, which $\mathrm{AO}_{1} \mathrm{~B}$ angle $=2$ pi was $\mathrm{ADB}$. Circle center coordinates and radius can be calculated by the following Eq. 1:

$$
\left\{\begin{array}{l}
\sqrt{\left(x_{1}-x_{a}\right)^{2}+\left(y_{1}-y_{a}\right)^{2}}=r_{1} \\
\sqrt{\left(x_{1}-x_{b}\right)^{2}+\left(y_{1}-y_{b}\right)^{2}}=r_{1} \\
\left(x_{a}-x_{b}\right)^{2}+\left(y_{a}-y_{b}\right)^{2}=2 r_{1}^{2}-2 r_{1}^{2} \cos A O_{1} B
\end{array}\right.
$$


Similarly, we can calculate the center of $\mathrm{O}, \mathrm{O}_{3}$ coordinate, and radius $\mathrm{r}_{2}, \mathrm{r}_{3}$ the trilateration calculated node D coordinates ( $\mathrm{x}, \mathrm{y}$ ), namely Eq.2:

$$
\left\{\begin{array}{l}
\sqrt{\left(x-x_{1}\right)^{2}+\left(y-y_{1}\right)^{2}}=r_{1} \\
\sqrt{\left(x-x_{2}\right)^{2}+\left(y-y_{2}\right)^{2}}=r_{2} \\
\sqrt{\left(x-x_{3}\right)^{2}+\left(y-y_{3}\right)^{2}}=r_{3}
\end{array}\right.
$$

The coordinates of the D (Eq. 3) of the node to be measured can be determined by the Eq.2:

$$
\left[\begin{array}{l}
x \\
x
\end{array}\right]=\left[\begin{array}{ll}
2\left(x_{1}-x_{3}\right) & 2\left(y_{1}-y_{3}\right) \\
2\left(x_{2}-x_{3}\right) & 2\left(y_{2}-y_{3}\right)
\end{array}\right]^{-1}\left[\begin{array}{l}
x_{1}^{2}-x_{3}^{2}+y_{1}^{2}-y_{3}^{2}+d_{3}^{2}-d_{1}^{2} \\
x_{2}^{2}-x_{3}^{2}+y_{2}^{2}-y_{3}^{2}+d_{3}^{2}-d_{2}^{2}
\end{array}\right]
$$

Multilateration known more than three beacon nodes coordinate information and beacon node to the unknown node distance information, using the distance formula between two points can be calculated the distance between unknown nodes and beacon nodes. Finally, by using the LS (Least Square), MLE (Maximum Likelihood Estimation) or MMSE(Minimum Mean Square Error) and for the coordinates of the unknown nodes.

Triangulation is the unknown nodes of the receiver antenna array to measure peripheral beacon signals sent by the incidence angle information and the angle information and the beacon nodes coordinate information. According to the coordinates of the Trilateration calculate the unknown node.

Maximum Likelihood Method principle as shown in Fig. 3.

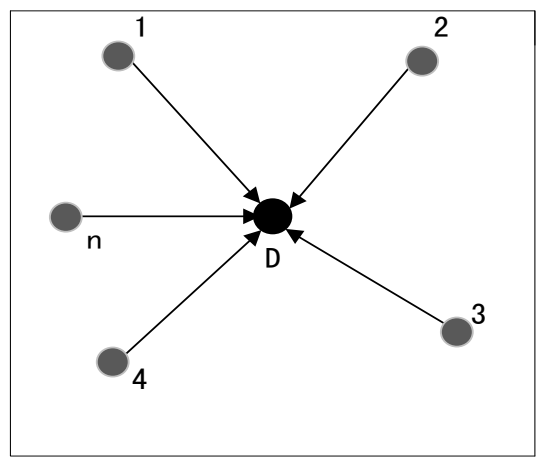

Figure 3. Maximum Likelihood Method

Known in the algorithm 1,2,3, The coordinates of $\mathrm{n}$ and other $\mathrm{n}$ beacon nodes are: $\left(x_{1}, y_{1}\right),\left(x_{2}, y_{2}\right),\left(x_{3}, y_{3}\right) \ldots\left(x_{n}, y_{n}\right)$.They are the distance to unknown node $\mathrm{D}$, respectively: $d_{1}$ 、 $d_{2}, d_{3} \ldots d_{n}$, assuming that the coordinates of the unknown node $\mathrm{D}$ are ${ }^{(x, y)}$ as follows Eq.4:

$$
\left\{\begin{array}{c}
\left(x_{1}-x\right)^{2}+\left(y_{1}-y\right)^{2}=d_{1} \\
\vdots \\
\left(x_{n}-x\right)^{2}+\left(y_{n}-y\right)^{2}=d_{n}
\end{array}\right.
$$

Starting from the first equation minus the last Eq.5:

$$
\left\{\begin{array}{c}
x_{1}^{2}-x_{n}{ }^{2}-2\left(x_{1}-x_{n}\right) x+y_{1}{ }^{2}-y_{n}{ }^{2}-2\left(y_{1}-y_{n}\right) y=d_{1}{ }^{2}-d_{n}{ }^{2} \\
\vdots \\
x_{n-1}{ }^{2}-x_{n}{ }^{2}-2\left(x_{n-1}-x_{n}\right) x+y_{n-1}^{2}-y_{n}^{2}-2\left(y_{n-1}-y_{n}\right) y=d_{n-1}{ }^{2}-d_{n}{ }^{2}
\end{array}\right.
$$


Eq. 5 can be expressed as: $A X=b$, in which: $A, b, X$, as the following Eq. 6 to Eq.8 as shown:

$$
\begin{aligned}
& A=\left[\begin{array}{cc}
2\left(x_{1}-x_{n}\right) & 2\left(y_{1}-y_{n}\right) \\
\vdots & \vdots \\
2\left(x_{n-1}-x_{n}\right) & 2\left(y_{n-1}-y_{n}\right)
\end{array}\right] \\
& b=\left[\begin{array}{c}
x_{1}^{2}-x_{n}^{2}+y_{1}^{2}-y_{n}^{2}+d_{n}^{2}-d_{1}^{2} \\
\vdots \\
x_{n-1}^{2}-x_{n}^{2}+y_{n-1}^{2}-y_{n}^{2}+d_{n}^{2}-d_{n-1}^{2}
\end{array}\right] \\
& X=\left[\begin{array}{c}
x \\
y
\end{array}\right]
\end{aligned}
$$

By using the maximum likelihood estimation method or the least square method, the coordinates of D can be estimated as Eq.9:

$$
\hat{X}=\left(A^{T} A\right)^{-1} A^{T} b
$$

\section{Summary}

This article mainly analyzes the localization algorithm based on distance, detailing the various algorithms, and the performance of various algorithms and application scope are introduced. Then form through the algorithm to a variety of network nodes and beacon node density, the localization error and positioning accuracy parameters respectively were compared, and analyses the advantages and disadvantages of each algorithm, and points out the problems existed in the different localization algorithm.

\section{References}

[1] AGRE J, CLARE L. An integrated architecture for cooperative sensing networks [J]. IEEE Computer Magazine, 2000, 33(5): 106-108.

[2] W. A Gardner, C. K Chen. Singal selective time difference of arrival estimation for Passive location. IEEE Transaction on Signal Processing, 2012, 2. 1168 1184.

[3] Huang Zhonghua, Li Shunhua. The Study on WSN Node Positioning Algorithm Improvement, 2012, International Conference on Computer Science and Service System, pp.1248-1249, 11-13 Aug. 2012.

[4] KANG HSEO G, LEE W J. Error compensation for css-based localization system[C]. Proceedings of the World Congress on Engineering and Computer Science, 2009.

[5] ANDREAS L, CHRISTIAN W. A comprehensive approach for optimizing To A-localization in harsh industrial environments[C]. Position Location and Navigation Symposium (PLANS), 2010 IEEE/ION, 2010. 516 525.

[6] ESTRIN D, GOVINDAN R, HEIDEMANN J. Next century challenges: scalable coordination in sensor networks [J]. Proceedings of the Fifth Annual International Conference on Mobile Computing and Networks (Mobi COM'99), Washington, USA, 1999: 263-270. 